\title{
Economical Doublet-Triplet Splitting and Strong Suppression of Proton Decay in $\mathrm{SO}(10)$
}

\author{
Z. Chacko and Rabindra N. Mohapatra \\ Department of Physics, University of Maryland, College Park, MD 20742, USA
}

\begin{abstract}
We present a new approach to realizing the Dimopoulos-Wiczek mechanism for doublet-triplet splitting in supersymmetric $\mathrm{SO}(10)$. The method can be used to achieve strong suppression of proton decay in a straightforward manner and is relatively economical; in particular the particle spectrum required for its implementation is consistent with the constraints from string compactification at KacMoody level two. We construct two examples of realistic models based on this idea. These models have no unwanted flat directions and do not make use of any nonrenormalizable operators to stabilize the vacuum thereby maintaining coupling constant unification as a prediction. The first model is characterised by a simple discrete symmetry that guarantees naturalness at the renormalizable level while also eliminating all potentially dangerous R-parity violating effects. The second model predicts the strong suppression of Higgsino mediated proton decay. In both models the light MSSM doublets emerge from two different 10's so that there is considerable flexibility in constructing realistic fermion masses and CKM angles.
\end{abstract}

UMD-PP-99-016

\section{INTRODUCTION}

One of the attractive features of the minimal supersymmetric extension of the standard model (MSSM) is that it leads to unification of the gauge couplings at a scale of $M_{U}$ about $\sim 2 \times 10^{16} \mathrm{GeV}$. This is very suggestive of the idea that all matter and forces of the standard model unify at the scale $M_{U}$ into a single unifying local symmetry [1,2]. The presence of supersymmetry provides the additional advantage that the large hierarchy between the weak and the GUT scale can be maintained order by order in perturbation theory thanks to the non-renormalization theorem of the superpotential.

A key problem of SUSY GUTs is how to split the weak MSSM doublets from the color triplet fields that accompany them as part of the representation of the GUT symmetry. This is an essential aspect of SUSY GUTs since both coupling constant unification and suppression of proton decay require that the MSSM doublets $H_{u}$ and $H_{d}$ must be at the weak scale whereas the triplets which mediate proton decay must have GUT scale mass. This is the famous doublet-triplet splitting (DTS) problem.

Another generic problem of SUSY GUT models such as $\mathrm{SU}(5)$ and $\mathrm{SO}(10)$ is that they lead to proton decay mediated by colored Higgsino fields. In the simplest models the predicted rate is consistent with observations only if the Higgsino masses are above the GUT scale [3] and this pushes these models to a very narrow range of the allowed parameter space. Alternatively, weak suppression can be obtained but only at the expense of large threshold effects [《]. It is therefore important to seek models that may ameliorate this problem.

There exist several ways to solve the DTS problem. It seems that the one most appropriate for the $\mathrm{SO}(10)$ model is the so called missing vev mechanism suggested by Dimopoulos and Wilczek(DW) [5] where one uses a 45-dim. Higgs which breaks the $\mathrm{SO}(10)$ symmetry down to $S U(2)_{L} \times S U(2)_{R} \times U(1)_{B-L} \times S U(3)_{c}$ group. The relevant vev is given by: $<\mathbf{4 5}>=\tau_{2} \times \operatorname{Diag}(a, a, a, 0,0)$. If one now couples this multiplet to two 10-dim. Higgs fields, it is easy to see that the MSSM doublet fields in the 10's remain massless. Since $\mathbf{4 5}$ is antisymmetric in the $\mathrm{SO}(10)$ indices, its coupling must involve two different 10's and all four of the standard model doublets in the two 10's remain light. Since this is two too many, one must add additional couplings which yield a mass term for one pair of doublets.

This method has the following serious difficulty. Once one includes other multiplets such as the $\mathbf{1 6}+\overline{\mathbf{1 6}}$ to break the $B-L$ symmetry the missing vev pattern essential to implement DTS is destabilized and the zeros in the $\mathbf{4 5}$ vev acquire GUT scale vevs; if one forbids the coupling of the 16's to the $\mathbf{4 5}$, then there will be additional massless Goldstone states associated with the breaking of a larger global symmetry of the superpotential which could upset coupling constant unification.

This and other difficulties in implementing the Dimopoulos-Wilczek mechanism were discussed in a very important paper by Babu and Barr [6] who showed how realistic $\mathrm{SO}(10)$ models could be constructed using this idea. They presented a model with a Higgs sector consisting of three 45's, two 54's, a $16+\overline{\mathbf{1 6}}$ and three 10's which could simultaneously solve the doublet-triplet splitting as well as the proton decay problem of the $\mathrm{SO}(10)$ models. The essence of their suggestion was that one of the $\mathbf{4 5}$ 's couples to the $\mathbf{1 6}+\overline{\mathbf{1 6}}$ pair while a second realizes doublet triplet splitting. The third $\mathbf{4 5}$ couples to the other two preventing any Goldstone states from appearing. It also helps suppress proton decay. This model however leads to unrealistic fermion masses and vanishing CKM angles since a 
single Yukawa coupling determines all of them. The question of naturalness in the sense of having a symmetry that prevents all unwanted terms in the superpotential was also not addressed. Subsequently alternative models based on the same idea have been constructed [7]. Unfortunately they also seem to require a similarly complicated Higgs sector to achieve DTS. A more economical solution to this problem would be attractive from the point of view of simplicity.

Further motivation for a more straightforward solution is provided by string theory. It is well known that string theories considerably restrict the number and nature of the gauge multiplets that survive to low energies. In particular the allowed $\mathrm{SO}(10)$ reprentations that can emerge as massless multiplets at the Kac-Moody level of two have been classified [8] [9]. While any number of vectors $(\mathbf{1 0}$ 's $)$, spinors $(\mathbf{1 6}+\overline{\mathbf{1 6}}$ 's $)$, and singlets are allowed the number of adjoints $(\mathbf{4 5}$ 's) is restricted to be at most two and the number of $\mathbf{5 4}$ 's not more than one. It is of interest to see whether realistic $\mathrm{SO}(10)$ models, and in particular doublet triplet splitting can be achieved within these constraints on the particle spectrum.

With this in mind there have been some earlier attempts to simplify the Higgs content of SO(10) models [11], [12], [13. However these models make use of non-renormalizable operators to realize the desired vacuum which implies the existence of Higgs fields with mass below the GUT scale, potentially upsetting the prediction of gauge coupling unification. In addition some of these models have flat directions which are undetermined at the classical level; hence their vacuum structure once SUSY is broken is unclear. Proton decay in these models is either unsuppressed or weakly suppressed.

In this paper, we present a relatively straightforward approach to realizing the Dimopoulos-Wilczek mechanism for doublet-triplet splitting in supersymmetric $\mathrm{SO}(10)$. The method is relatively economical; in particular the particle spectrum required for its implementation is consistent with the above mentioned constraints from string theory. Specifically the minimal field content consists of one $\mathbf{5 4}$ (denoted by $S$ ), two $\mathbf{4 5}$ 's $(A, \bar{A})$, two $\mathbf{1 0}$ 's $\left(H_{1,2}\right)$ and a $\mathbf{1 6}+\mathbf{1 6}$ pair (denoted by $C+\bar{C}$ ). The essence of our idea is that one $\mathbf{4 5}(\bar{A})$ acquires the Dimopoulos-Wilczek pattern of vevs while the other $(A)$ couples to the $\mathbf{1 6}+\mathbf{1 6}$. Since both these fields couple to the $\mathbf{5 4}$ there are no unwanted Goldstone modes. We construct two examples of realistic models based on this scheme. The models have no flat directions (apart from the light doublets) and do not use any nonrenormalizable terms to determine the desired vacuum, thereby maintaining coupling constant unification as a prediction. The first model is characterised by a simple discrete symmetry which guarantees naturalness at the renormalizable level while also eliminating all potentially dangerous R-parity violating effects and is described in sec. II. The second model predicts the strong suppression of Higgsino mediated proton decay along with doublet-triplet splitting. This is described in sec. III. Realistic fermion masses and CKM angles also arise very easily in both models essentially because the $H_{u}$ and $H_{d}$ of the MSSM emerge from two different 10's as suggested in [13]. As a result of this the up and the down sector mass matrices arise from different Yukawa couplings and therefore the CKM angles do not vanish.

The next two sections are devoted to a detailed analysis of the supersymmetric vacua of the two models that we present as well as a demonstration of doublet triplet splitting and proton decay in the case of the second model.

\section{MODEL I:}

\section{A. Symmetry Breaking Sector}

The part of the superpotential relevant for the breaking of $\mathrm{SO}(10)$ down to the MSSM is given by:

$$
\begin{array}{r}
W_{H}=M_{S} S^{2}+\lambda_{S} S^{3}+M_{A}\left[A^{2}+\beta \bar{A}^{2}\right] \\
+\lambda_{1} S\left[A^{2}+\alpha \bar{A}^{2}\right]+\gamma \operatorname{Tr} S \\
+2 M_{C} C \bar{C}-\lambda_{C} C A \bar{C}
\end{array}
$$

where the various fields transform under $\mathrm{SO}(10)$ as explained in the introduction. We further impose a discrete $Z_{4}$ symmetry on the theory which (as will be clear later) guarantees naturalness at the renormalizable level. The transformations of the various fields under this symmetry are shown in Table I. $\gamma$ is the Lagrange multiplier that imposes the trace condition. We can assume the following vacuum configurations for the various fields:

$$
\begin{array}{r}
<S>=I_{2} \times \operatorname{Diag}\left(s_{1}, s_{1}, s_{1},-\frac{3}{2} s_{1},-\frac{3}{2} s_{1},\right) \\
<A>=\tau_{2} \times \operatorname{Diag}(a, a, a, b, b) \\
<\bar{A}>=\tau_{2} \times \operatorname{Diag}(\bar{a}, \bar{a}, \bar{a}, \bar{b}, \bar{b}) \\
<C_{\nu^{c}}>=<\bar{C}_{\nu^{c}}>=v ;
\end{array}
$$


The equality of the vev's of $C$ and $\bar{C}$ is dictated by the vanishing of the D-terms at the GUT scale needed to mainatin supersymmetry down to the electroweak scale.

Setting the various $F$-terms to zero, we get the following equations. For $F_{S}=0$, we have:

$$
5 M_{S} s_{1}-\frac{15}{4} \lambda_{S} s_{1}^{2}+\lambda_{1}\left[a^{2}-b^{2}\right]+\alpha \lambda_{1}\left[\left(\bar{a}^{2}-\bar{b}^{2}\right]=0\right.
$$

From the equation of motion for $\bar{A}$ we get

$$
\begin{aligned}
\left(M_{A} \beta+\lambda_{1} s_{1} \alpha\right) \bar{a} & =0 \\
\left(M_{A} \beta-\frac{3}{2} \lambda_{1} s_{1} \alpha\right) \bar{b} & =0
\end{aligned}
$$

The above two equations have three solutions: (i) $\bar{a}=0$ and $\bar{b}=0$; (ii) $\bar{a} \neq 0$, which means that $s_{1}=-\frac{\beta M_{A}}{\lambda_{1} \alpha}$ and $\bar{b}=0$ and (iii) $\bar{b} \neq 0$ which means $s_{1}=\frac{2 M_{A} \beta}{3 \lambda_{1} \alpha}$ and $\bar{a}=0$. All of these are inequivalent vacuua. We will assume that we are in the second one so that $\bar{b}=0$ and the DW mechanism is realized.

The equations of motion for $A$ yield (using the solution for $s_{1}$ from above)

$$
\begin{aligned}
& a M_{A}\left(1-\frac{\beta}{\alpha}\right)+\frac{1}{2} \lambda_{C} v^{2}=0 \\
& b M_{A}\left(1+\frac{3 \beta}{2 \alpha}\right)+\frac{1}{2} \lambda_{C} v^{2}=0
\end{aligned}
$$

The $F_{C}=F_{\bar{C}}=0$ conditions yield

$$
M_{C}+\lambda_{C}[3 a+2 b]=0
$$

Using the above Eq.(3), (5), (6) and (7), we can determine the values of $b, a, \bar{a}$ and $v$, in terms of the parameters in the superpotential. Note that $\bar{A}$ has the Dimopoulos-Wilczek pattern of vevs. Since both this field and the $A$ field which couples to the $\mathbf{1 6}$ 's couple to the $\mathbf{5 4}$ there are no unwanted Goldstone modes.

\section{B. Doublet-triplet splitting and Fermion masses}

There are many ways to use the missing vev pattern described above to obtain the MSSM doublets from the GUT scale physics. The simplest is to use the superpotential

$$
W_{1}=\lambda_{H} H_{1} \bar{A} H_{2}+C C\left(p H_{1}+q H_{2}\right)+\bar{C} \bar{C}\left(p^{\prime} H_{1}+q^{\prime} H_{2}\right)
$$

However we would prefer to implement the DTS in such a way that it is consistent with the discrete $Z_{4}$ symmetry that ensures the naturalness of the theory. To this end we introduce an additional $(\mathbf{1 6 , 1 6})$ pair, $P$ and $\bar{P}$. We can self consistently assume that they have zero vev in the supersymmetric limit. Then the part of the superpotential that generates the light MSSM doublets $W_{D T S}$ is given below

$$
W_{D T S}=\lambda_{H} H_{1} \bar{A} H_{2}+C P\left(p H_{1}+q H_{2}\right)+\bar{C} \bar{P}\left(p^{\prime} H_{1}+q^{\prime} H_{2}\right)+\lambda_{P} P \bar{A} \bar{P}
$$

The role of the various terms in $W_{D T S}$ is not hard to understand. At the GUT scale, our model has four up-type and four down type MSSM doublets; of these the doublets belonging to $C, \bar{C}$ become superheavy due to the Higgs sector described in the previous section leaving us with three up and down Higgs doublets i.e. $\left(H_{1 u}, H_{2 u}, \bar{P}_{u}\right)$ and $\left(H_{1 d}, H_{2 d}, P_{d}\right)$. It is clear from Eq.(9) that $P_{d}$ and one linear combination of $H_{1 u}$ and $H_{2 u}$ pair up to become superheavy and $\bar{P}_{u}$ and a linear combination of $H_{1 d}$ and $H_{2 d}$ also pair up and decouple. The light MSSM Higgs doublets therefore are the orthogonal linear combinations. On the other hand, the triplets present in $H_{1,2}$ become superheavy due to the $H_{1} H_{2} A$ term in the $W_{D T S}$, where as those in $P$ and $\bar{P}$ become superheavy by the similar term. The triplets in $C$ and $\bar{C}$ also become superheavy due to the Higgs sector described in the previous section. Thus there are no light triplets below GUT scale. This method of using the 16's to split doublets was first noted in Ref. [13] and was subsequently used in [12]. It is straightforward to verify that the superpotential for the Higgs fields $W_{H}+W_{D T S}$ has the most general form compatible with the gauge and discrete symmetries at the renormalizable level. 
In order to discuss the fermion masses in this model, we must write down the Yukawa part of the superpotential which is invariant under the $Z_{4}$ symmetry. Using the transformation properties in of the fields under these symmetries given in table I, we get:

$$
\begin{array}{r}
W_{Y}=h_{a b} \psi_{a} \psi_{b} H_{2}+h_{a b}^{\prime} \psi_{a} \psi_{b} H_{1} \\
+h_{a b}^{\prime \prime} \psi_{a} \psi_{b} A H / M+\ldots
\end{array}
$$

Using this one can construct realistic fermion masses. Note that the Yukawa matrices are arbitrary in generation space which allows for many more possibilities for mass textures.

It is well known that in $\mathrm{SO}(10)$ models where the $B-L$ symmetry is broken by $\mathbf{1 6}$ vevs, operators like $\psi \psi \psi C / M_{P l}$ lead after symmetry breaking to baryon and lepton number violating (R-parity violating) terms with strengths of order $10^{-2}$, in strong disagreement with the existing limits on such couplings from proton decay etc. 14. It is worth pointing out that the same discrete symmetry that led to a natural realization of the doublet-triplet splitting also forbids all dangerous R-parity violating terms. The lowest order term allowed by the symmetry is of the form $\psi^{6} C^{2} / M_{P}^{5}$. This leads to a generic sixth order term in quark fields such as $u^{c} u^{c} \tilde{d}^{c} \tilde{d}^{c} \tilde{d}^{c} \tilde{d}^{c}$ with a strength of order $10^{-58} \mathrm{GeV}^{-3}$ and has no observable effect.

In this model, in the limit of $p, p^{\prime}, q, q^{\prime}, h_{a b}^{\prime}=0$, Higgsino mediated contributions to proton decay vanish. However in this limit, CKM angles also vanish and there is an extra pair of MSSM doublets. Therefore, it is possible to get additional suppression over the minimal SU(5) prediction for proton decay as long as the extra doublet pair is slightly below the GUT scale. This would generate new threshold corrections which should be taken into account in the discussion of unification.

\section{MODEL II:}

\section{A. Symmetry Breaking Sector}

As demonstrated in [6], strong suppression of proton decay can be realized in supersymmetric $\mathrm{SO}(10)$ if a 45 has the complimentary pattern of vev's to the Dimopoulos-Wilczek pattern, ie $\tau_{2} \times \operatorname{Diag}(0,0,0, a, a)$. In this section we present the sector of the model which breaks SO(10) down to the MSSM. We will show that in this process, this pattern of vevs is generated for the field $A$. The field $\bar{A}$ will be shown to retain the Dimopoulos-Wilczek form, thereby realizing doublet-triplet splitting. The relevant part of the superpotential is given by

$$
\begin{array}{r}
W_{S B}^{1}=M_{1} S^{2}+\lambda S^{3}+S A \bar{A}+\frac{3}{2} Y A \bar{A} \\
+\gamma \operatorname{Tr} S+M_{0} A^{2}-\beta C A \bar{C}+2 M_{2} C \bar{C}
\end{array}
$$

where $Y$ is an $\mathrm{SO}(10)$ singlet field, and the other fields transform as before. We have scaled all fields so as to set some couplings to one. We can assume the following vacuum configurations for the various fields:

$$
\begin{array}{r}
<S>=I_{2} \times \operatorname{Diag}\left(s_{1}, s_{2}, s_{3}, s_{4}, s_{5}\right) \\
<A>=\tau_{2} \times \operatorname{Diag}\left(a_{1}, a_{2}, a_{3}, a_{4}, a_{5}\right) \\
<\bar{A}>=\tau_{2} \times \operatorname{Diag}\left(\overline{a_{1}}, \overline{a_{2}}, \overline{a_{3}}, \overline{a_{4}}, \overline{a_{5}}\right) \\
<C_{\nu^{c}}>=<\overline{C_{\nu^{c}}}>=v
\end{array}
$$

The equations for the vanishing $F$-terms at the GUT scale are:

$$
\begin{gathered}
\Sigma_{i} a_{i} \overline{a_{i}}=0 \\
s_{i} \bar{a}_{i}+\frac{3}{2} y \bar{a}_{i}+2 M_{0} a_{i}+\beta v^{2}=0 \\
3 \lambda s_{i}^{2}+2 M_{1} s_{i}+\bar{a}_{i} a_{i}+\gamma=0 \\
s_{i} a_{i}+\frac{3}{2} y a_{i}=0
\end{gathered}
$$




$$
M_{2}+\beta \Sigma_{i} a_{i}=0
$$

All vev's are given in obvious notation. We are interested in the ground state for which $a_{4}=a_{5} \neq 0, a_{\alpha}=0$ (for $\alpha=1,2,3), \overline{a_{\alpha}} \neq 0$ and $\overline{a_{4}}=\overline{a_{5}}=0$. Let us start with the first two equations and use the $F$-term conditions above to show that the second two equations follow. From the above equations it is easy to see that the first two conditions (i.e. $a_{4,5} \neq 0$ and $a_{\alpha}=0$ ) imply that $s_{4}=s_{5}=-\frac{3}{2} y$ leaving $s_{\alpha}$ undetermined. Eq. (15) and the tracelessness of $S$ then imply that $s_{\alpha}$ are all equal to $y$. Using Eq.(15), we get $a_{4}=a_{5}=-\frac{\beta v^{2}}{2 M_{0}}$. Using Eq. (14) and (15), it is easy to see that $\overline{a_{4}}=\overline{a_{5}}=0$ and all $\overline{a_{\alpha}}$ are equal as desired. The Equation (15) for $F_{S}=0$ then leads to two solutions for $s_{i}$ whose sum is equal to $-\frac{2 M_{1}}{3 \lambda}$ thus solving for $y$ and $s_{i}$. The Eq.(17) for $F_{C}=0$ then determines $a_{4}$ and thus $v$; Eq. (14) then determines $\bar{a}$. Hence $A$ and $\bar{A}$ have the desired pattern of vevs $\tau_{2} \times D i a g(0,0,0, a, a)$ and $\tau_{2} \times \operatorname{Diag}(\bar{a}, \bar{a}, \bar{a}, 0,0)$ respectively.

The model as we have presented above has a pair of massless singly charged fields which arise from the $(\mathbf{1}, \mathbf{3}, \mathbf{1})$ (under $\left.S U(2)_{L} \times S U(2)_{R} \times S U(4)_{c}\right)$ component of the $\bar{A}$ field. This problem however can be easily cured without effecting the rest of the discussion by introducing an extra pair of $\mathbf{1 6}+\overline{\mathbf{1 6}}$ (denoted by $\mathbf{P}+\overline{\mathbf{P}}$ as before) and a pair of singlet fields $\mathbf{T}+\overline{\mathbf{T}}$. We then add to the above superpotential the following piece:

$$
W^{(3)}=C \bar{A} \bar{P}+\bar{C} \bar{A} P+C T \bar{P}+\bar{C} \bar{T} P+M^{\prime} P \bar{P}
$$

Note that minima equations for $T$ and $\bar{T}$ imply that $P$ and $\bar{P}$ have vanishing vev's but $T$ and $\bar{T}$ have nonzero vevs. It is then easy to see that the massless fields of $\bar{A}$ are now lifted to GUT scale as they pair up with right handed doublets from $P$ and $\bar{P}$.

\section{B. Doublet-Triplet spltting, Strong Suppression of Proton Decay and Fermion Masses in Model II}

It was pointed out in [6] that by increasing the number of 10-dim. representations to three, one can obtain a strong suppression of proton decay, if the following superpotential $\left(W_{B B}\right)$ is chosen:

$$
W_{B B}=H_{1} \bar{A} H_{2}+H_{2} A H_{3}+M_{3} H_{3}^{2}
$$

The problem with this model lies however with the pattern of fermion masses it leads to. It is easy to see that the light MSSM doublets in this model belong to the same 10; as a result, one gets vanishing quark mixing angles. This problem is solved if we have four 10-dim. fields $H_{1,2,3,4}$ and we choose the following superpotential to implement both doublet-triplet splitting and achieve strong suppression of proton decay:

$$
\begin{array}{r}
W_{D T S}=H_{1} \bar{A} H_{2}+\left(H_{1}+H_{2}\right) A\left(H_{3}+H_{4}\right)+H_{3} \bar{A} H_{4} \\
+C C H_{1}+\bar{C} \bar{C} H_{3}
\end{array}
$$

From $W_{D T S}$ it is clear that there is exactly one pair of massless MSSM doublets. This can be seen more explicitly by writing down the mass matrix for the doublet fermions in the $\mathbf{1 0}$ 's, the $\mathbf{C}+\overline{\mathbf{C}}$ and the $\mathbf{P}+\overline{\mathbf{P}}$ :

$$
M_{D}=\left(\begin{array}{llllll}
H_{1 u} & H_{2 u} & H_{3 u} & H_{4 u} & \bar{C}_{u} & \bar{P}_{u}
\end{array}\right)\left(\begin{array}{cccccc}
0 & 0 & a & a & v & 0 \\
0 & 0 & a & a & 0 & 0 \\
a & a & 0 & 0 & 0 & 0 \\
a & a & 0 & 0 & 0 & 0 \\
0 & 0 & v & 0 & 2 M_{2} & 12 \bar{a} \\
0 & 0 & 0 & 0 & 12 \bar{a} & M^{\prime}
\end{array}\right)\left(\begin{array}{c}
H_{1 d} \\
H_{2 d} \\
H_{3 d} \\
H_{4 d} \\
C_{d} \\
P_{d}
\end{array}\right)
$$

where we have denoted the vev of $A$ by $a$ and that of $\bar{A}$ by $\bar{a}$. We remind the reader that $<A>=\tau_{2} \times D i a g(0,0,0, a, a)$ and $\langle\bar{A}\rangle=\operatorname{Diag}(\bar{a}, \bar{a}, \bar{a}, 0,0)$. The light doublets are then given by

$$
\begin{aligned}
H_{u} & =\left[\left(H_{3}\right)_{u}-\left(H_{4}\right)_{u}\right] / \sqrt{2} \\
H_{d} & =\left[\left(H_{1}\right)_{d}-\left(H_{2}\right)_{d}\right] / \sqrt{2}
\end{aligned}
$$

To see the color triplet masses, we write their mass matrix as follows:

$$
M_{T}=\left(\begin{array}{llllll}
\xi_{1} & \xi_{4} & \xi_{2} & \xi_{3} & \xi_{C} & \xi_{P}
\end{array}\right)\left(\begin{array}{cccccc}
\bar{a} & 0 & 0 & 0 & 0 & 0 \\
0 & \bar{a} & 0 & 0 & 0 & 0 \\
0 & 0 & \bar{a} & 0 & 0 & 0 \\
0 & 0 & 0 & \bar{a} & v & 0 \\
0 & 0 & v & 0 & 4 M_{2} & 8 \bar{a} \\
0 & 0 & 0 & 0 & 8 \bar{a} & M^{\prime}
\end{array}\right)\left(\begin{array}{c}
\bar{\xi}_{2} \\
\bar{\xi}_{3} \\
\bar{\xi}_{1} \\
\bar{\xi}_{4} \\
\bar{\xi}_{\bar{C}} \\
\bar{\xi}_{\bar{P}}
\end{array}\right)
$$


First it is clear from this equation that all color triplets have GUT scale mass. Moreover note that $\xi_{1}$ and $\xi_{4}$ do not connect to $\bar{\xi}_{1}$ and $\bar{\xi}_{4}$. As a result, if we choose the fermion Yukawa couplings to be of the form

$$
W_{Y}=h_{u} \psi \psi H_{1}+h_{d} \psi \psi H_{4}
$$

there are no dimension five Higgsino mediated proton decay operators induced by the color triplet fields. Furthermore, we the see that the up and the down type fermion masses arise from two different couplings and we therefore have freedom to construct realistic fermion mass matrices. We can correct for the bad mass relation $m_{e} / m_{\mu}=m_{d} / m_{s}$ by adding higher dimensional terms of the form $\psi \psi A \bar{A} H_{1}$ etc as explained in [13].

It is conceivable that the form of the superpotential or some generalization of it is dictated by a set of discrete symmetries; however, we have not made any attempt to find them here.

\section{CONCLUSION}

In conclusion, we have presented a method of implementing doublet triplet splitting in supersymmetric $\mathrm{SO}(10)$ in a simple and economical way using the field content dictated by fermionic compactification of superstrings. Two realistic models have been constructed using this idea. The models have no unwanted flat directions, maintain coupling constant unification as a prediction and give rise to realistic fermion masses. Strong suppression of Higgsino mediated proton decay emerges as a prediction of one of the models.

This work is supported by the National Science Foundation under grant no. PHY-9802551.

[1] J. C. Pati and A. Salam, Phys. Rev. D 10, 275 (1974); H. Georgi and S. L. Glashow,Phys. Rev. Lett.32, 438 (1974); H. Georgi, H. Quinn and S. Weinberg, Phys. Rev. Lett. 33, 451 (1974).

[2] For a recent review, see R. N. Mohapatra, TASI97 lectures, hep-ph/9801235.

[3] R. Arnowitt and P. Nath, Phys. Rev. D 49, 1479 (1994); J. Hisano, H. Murayama and T. Yanagida, Tohuku Univ. preprint TU-400 (1992).

[4] S. Urano and R. Arnowitt, hep-ph/9611389.

[5] S. Dimopoulos and F. Wilczek, Preprint NSF-ITP-82-07.

[6] K. S. Babu and S.M. Barr, Phys. Rev. D 48, 5354 (1993).

[7] K. S. Babu and S.M. Barr, Phys. Rev. D 50, 3529 (1994).

[8] S.Choudhuri, S.Chung and J.Lykken, FERMILAB-PUB-94/137-T (1994)

[9] G.Cleaver,Ohio State Preprint OHSTPY-HEP-T-94-007 (1994)

[10] Keith R. Dienes, Nucl. Phys. B 488, 141 (1997)

[11] K. S. Babu and S.M. Barr, Phys. Rev. D 51, 2463 (1995).

[12] S.M. Barr and S. Raby, Phys. Rev. Lett 79, 4748 (1997); C. Albright and S. Barr, hep-ph/9712488.

[13] K. S. Babu and R. N. Mohapatra, Phys. Rev. Lett. 74, 2418 (1995).

[14] For a recent review, see G. Bhattacharyya, Nucl. Phys. Proc. Supp., 52A, 83 (1994).

\begin{tabular}{|c||c||}
\hline Fields & $Z_{4}$ \\
\hline$H_{1}$ & $i H_{1}$ \\
$H_{2}$ & $i H_{2}$ \\
$A$ & $A$ \\
$\bar{A}$ & $-\bar{A}$ \\
$C$ & $C$ \\
$\bar{C}$ & $\bar{C}$ \\
$\psi_{a}$ & $e^{\frac{i \pi}{4}} \psi_{a}$ \\
$P$ & $-i P$ \\
$\bar{P}$ & $-i \bar{P}$ \\
\hline
\end{tabular}

TABLE I. Transformation of the different fields under the discrete symmetry $Z_{4}$. 